\title{
Supersymmetric left-right model with radiative neutrino mass and multipartite dark matter
}

\author{
Subhaditya Bhattacharya ${ }^{\mathrm{a}},{\text { Ernest } \mathrm{Ma}^{\mathrm{b}} \text {, Daniel Wegman }}^{\mathrm{c}}$ \\ Department of Physics and Astronomy, University of California, Riverside, CA 92521, USA
}

Received: 19 December 2013 / Accepted: 9 May 2014 / Published online: 4 June 2014

(C) The Author(s) 2014. This article is published with open access at Springerlink.com

\begin{abstract}
We propose a unified extension of the standard model with three types of coexisting dark matter, motivated by three well-studied scenarios: (1) supersymmetry with $R$-parity conservation, (2) left-right symmetry where the $S U(2)_{R}$ doublet is not $(v, e)_{R}$ but $(n, e)_{R}$ where $n$ is a dark-matter candidate, and (3) a one-loop radiative neutrino mass model where the particles in the loop have an odd $Z_{2}$ symmetry, the lightest of which is also a dark-matter candidate. Whereas there are many new particles in this model, the gauge couplings get unified in the same way as in the minimal supersymmetric standard model (MSSM). Since there are at least three dark-matter candidates, each one's share of the total relic density of the Universe is adjustable, allowing for a much larger parameter space for the MSSM candidate itself. Our main focus is a careful study of a possible specific multipartite dark-matter scenario in this context.
\end{abstract}

\section{Introduction}

To understand dark matter in the context of extensions of the standard model of particle interactions, there are many avenues. Supersymmetry with $R$-parity conservation is the most common approach. Two other well-motivated scenarios have also been proposed in recent years. One is the idea of radiative neutrino mass induced by dark matter. The simplest such one-loop mechanism was proposed by one of us in 2006 [1]. It has been called "scotogenic", from the Greek "scotos" meaning darkness. This proposal has been studied and extended in a number of subsequent papers [211]. Another is to have a left-right extension where the neutral component $n$ of the $S U(2)_{R}$ doublet $(n, e)_{R}$ is the dark matter [12-17].

\footnotetext{
a e-mail: subhaditya123@gmail.com

be-mail: ma@phyun8.ucr.edu

c e-mail: wegman.daniel@gmail.com
}

It was pointed out [16] that with the addition of new supermultiplets, the dark left-right model is unifiable with all gauge couplings converging at an energy scale of about $10^{16} \mathrm{GeV}$. These additional particles turn out to be exactly what are required for radiative neutrino masses in the scotogenic model [1]. Hence an opportunity exists for merging all three mechanisms for dark matter in the context of a supersymmetric unified theory with radiative neutrino mass. In this paper we will focus mainly on the dark-matter (DM) phenomenology of this comprehensive model. Note that there are at least three dark-matter candidates. Each one's share of the total relic density of the Universe is adjustable. This frees up the usual parameter space of the minimal supersymmetric standard model (MSSM) due to this constraint. It allows collider signatures of supersymmetry in an otherwise excluded region.

\section{Model}

Consider the gauge group $S U(3)_{C} \times S U(2)_{L} \times S U(2)_{R} \times$ $U(1)$. A new global $U(1)$ symmetry $S$ is imposed so that the spontaneous breaking of $S U(2)_{R} \times S$ will leave the combination $S^{\prime}=S+T_{3 R}$ unbroken. Under $S U(3)_{C} \times S U(2)_{L} \times$ $S U(2)_{R} \times U(1) \times S \times M \times H$, where $M$ and $H$ are discrete $Z_{2}$ symmetries, with the usual $R$ parity of the MSSM given by $R \equiv M H(-1)^{2 j}$, the superfields transform as shown in Table 1. Because of supersymmetry, the Higgs sector is doubled, in analogy to the transition from the standard model (SM) to the MSSM. Another set of Higgs doublet superfields, $\eta$, and a new set of charged and neutral Higgs singlet superfields, $\zeta$, are added to obtain gauge-coupling unification, as well as radiative seesaw neutrino masses.

The superpotential of the model reads

$$
\begin{aligned}
W= & -\mu_{L} \Phi_{L 1} \Phi_{L 2}-\mu_{R} \Phi_{R 1} \Phi_{R 2} \\
& -\mu_{\Delta} \operatorname{Tr}\left(\Delta_{1} \Delta_{2}\right)-\mu_{N} N N
\end{aligned}
$$


Table 1 Particle content of proposed model

\begin{tabular}{lllll}
\hline Superfield & $\begin{array}{l}S U(3)_{C} \times S U(2)_{L} \\
\times S U(2)_{R} \times U(1)\end{array}$ & $S$ & $M$ & $H$ \\
\hline$\psi=(v, e)$ & $(1,2,1,-1 / 2)$ & 0 & - & + \\
$\psi^{c}=\left(e^{c}, n^{c}\right)$ & $(1,1,2,1 / 2)$ & $-1 / 2$ & - & + \\
$N$ & $(1,1,1,0)$ & 0 & - & - \\
$n$ & $(1,1,1,0)$ & 1 & - & + \\
$Q=(u, d)$ & $(3,2,1,1 / 6)$ & 0 & - & + \\
$Q^{c}=\left(h^{c}, u^{c}\right)$ & $\left(3^{*}, 1,2,-1 / 6\right)$ & $1 / 2$ & - & + \\
$d^{c}$ & $\left(3^{*}, 1,1,1 / 3\right)$ & 0 & - & + \\
$h$ & $(3,1,1,-1 / 3)$ & -1 & - & + \\
$\Delta_{1}$ & $(1,2,2,0)$ & $1 / 2$ & + & + \\
$\Delta_{2}$ & $(1,2,2,0)$ & $-1 / 2$ & + & + \\
$\Phi_{L 1}$ & $(1,2,1,-1 / 2)$ & 0 & + & + \\
$\Phi_{L 2}$ & $(1,2,1,1 / 2)$ & 0 & + & + \\
$\Phi_{R 1}$ & $(1,1,2,-1 / 2)$ & $-1 / 2$ & + & + \\
$\Phi_{R 2}$ & $(1,1,2,1 / 2)$ & $1 / 2$ & + & + \\
$\eta_{L 1}$ & $(1,2,1,-1 / 2)$ & 0 & + & - \\
$\eta_{L 2}$ & $(1,2,1,1 / 2)$ & 0 & + & - \\
$\eta_{R 1}$ & $(1,1,2,-1 / 2)$ & $1 / 2$ & + & - \\
$\eta_{R 2}$ & $(1,1,2,1 / 2)$ & $-1 / 2$ & + & - \\
$\zeta_{1}$ & $(1,1,1,-1)$ & 0 & + & - \\
$\zeta_{2}$ & $(1,1,1,1)$ & 0 & + & - \\
$\zeta_{3}$ & $(1,1,1,0)$ & 0 & + & - \\
\hline & & & &
\end{tabular}

$$
\begin{aligned}
& -\mu_{L 2} \eta_{L 1} \eta_{L 2}-\mu_{R 2} \eta_{R 1} \eta_{R 2}-\mu_{s 12} \zeta_{1} \zeta_{2}-\mu_{S 3} \zeta_{3} \zeta_{3} \\
& +f_{1} \Phi_{L 1} \Delta_{2} \Phi_{R 2}+f_{2} \Phi_{L 2} \Delta_{1} \Phi_{R 1} \\
& +f_{3} \eta_{L 1} \Delta_{1} \eta_{R 2}+f_{4} \eta_{L 2} \Delta_{2} \eta_{R 1}+f_{5} \Phi_{L 1} \eta_{L 1} \zeta_{2} \\
& +f_{6} \Phi_{R 1} \eta_{R 1} \zeta_{2}+f_{7} \Phi_{L 2} \eta_{L 2} \zeta_{1}+f_{8} \Phi_{R 2} \eta_{R 2} \zeta_{1} \\
& +f_{9} \Phi_{L 1} \eta_{L 2} \chi_{3}+f_{10} \Phi_{L 2} \eta_{L 1} \zeta_{3} \\
& +f_{11} \psi \Delta_{1} \psi^{c}+f_{12} Q \Delta_{2} Q^{c}+f_{13} Q \Phi_{L 1} d^{c} \\
& +f_{14} n \psi^{c} \Phi_{R 1}+f_{15} h Q^{c} \Phi_{R 2} \\
& +f_{16} \psi N \eta_{L 2}+f_{17} \psi^{c} N \eta_{R 1} .
\end{aligned}
$$

The symmetry $S \times M \times H$ is used here to distinguish $\psi, \Phi_{L 1}$, and $\eta_{L 1}$ from one another, as well as $\psi^{c}, \Phi_{R 2}$, $\eta_{R 2}$, and $N, n, \zeta_{3}$. There are seven bilinear terms with coefficients $\mu$ and 17 trilinear terms with coefficient $f$ allowed by $S \times M \times H$.

Hence $m_{e}$ comes from the $I_{3 L}=1 / 2$ and $I_{3 R}=-1 / 2$ component of $\Delta_{1}$, i.e. $\delta_{11}^{0}\left(\left\langle\delta_{11}^{0}\right\rangle=u_{1}\right)$ with $S^{\prime}=1 / 2-$ $1 / 2=0, m_{u}$ from the $I_{3 L}=-1 / 2$ and $I_{3 R}=1 / 2$ component of $\Delta_{2}$, i.e. $\delta_{22}^{0}\left(\left\langle\delta_{22}^{0}\right\rangle=u_{4}\right)$ with $S^{\prime}=-1 / 2+1 / 2=0$, $m_{d}$ from $\phi_{L 1}^{0}\left(\left\langle\phi_{L 1}^{0}\right\rangle=v_{L 1}\right), m_{n}$ from $\phi_{R 1}^{0}\left(\left\langle\phi_{R 1}^{0}\right\rangle=v_{R 1}\right)$, and $m_{h}$ from $\phi_{R 2}^{0}\left(\left\langle\phi_{R 2}^{0}\right\rangle=v_{R 2}\right)$. Note that $\phi_{L 2}^{0}\left(\left\langle\phi_{L 2}^{0}\right\rangle=\right.$ $\left.v_{L 2}\right)$ does not contribute to fermion masses, but it is involved in the scalar and vector masses. This structure guarantees the absence of tree-level flavor-changing neutral currents, which was the original motivation of this proposal and allows the
$S U(2)_{R}$ breaking scale to be a few $\mathrm{TeV}$. In the conventional $S U(2)_{R}$ model, this would not be possible without fine tuning.

The scalar sector is extremely complex, containing 26 neutral and 28 charged scalars. The mass matrices are non-trivial, and the following bases are decoupled from each other:

1. $\left\{\Re\left(\phi_{L 1}\right), \Re\left(\phi_{L 2}\right), \Re\left(\phi_{R 1}\right), \Re\left(\phi_{R 2}\right), \Re\left(\delta_{11}\right), \Re\left(\delta_{22}\right)\right\}$,

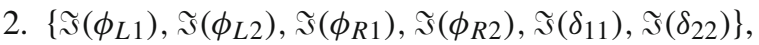

3. $\left\{\Re\left(\eta_{L 1}\right), \Re\left(\eta_{L 2}\right), \Re\left(\zeta_{3}\right)\right\}$,

4. $\left\{\Im\left(\eta_{L 1}\right), \Im\left(\eta_{L 2}\right), \Im\left(\zeta_{3}\right)\right\}$,

5. $\left\{\Re\left(\delta_{12}\right), \Re\left(\delta_{21}\right)\right\}$,

6. $\left\{\Im\left(\delta_{12}\right), \Im\left(\delta_{21}\right)\right\}$,

7. $\left\{\Re\left(\eta_{R 1}\right), \Re\left(\eta_{R 2}\right)\right\}$,

8. $\left\{\Im\left(\eta_{R 1}\right), \Im\left(\eta_{R 2}\right)\right\}$.

For the charged Higgs the decoupled bases are:

1. $\left\{\phi_{L 1}^{ \pm}, \phi_{L 2}^{ \pm}, \delta_{11}^{ \pm}, \delta_{22}^{ \pm}\right\}$,

2. $\left\{\phi_{R 1}^{ \pm}, \phi_{R 2}^{ \pm}, \delta_{12}^{ \pm}, \delta_{21}^{ \pm}\right\}$,

3. $\left\{\eta_{L 1}^{ \pm}, \eta_{L 2}^{ \pm}, \eta_{R 1}^{ \pm}, \eta_{R 2}^{ \pm}, \zeta_{1}^{ \pm}, \zeta_{2}^{ \pm}\right\}$.

\section{Radiative seesaw neutrino masses}

Since the neutrino $v$ does not couple to $N$ through $\Phi_{L 2}$, it has no tree-level mass. However, the $v N \eta_{L 2}^{0}$ and $\phi_{L 1}^{0} \eta_{L 2}^{0} \zeta_{3}$ couplings and the allowed Majorana masses for $N$ and $\zeta_{3}$ will generate one-loop radiative seesaw neutrino masses, as shown in Fig. 1.

The loop can be calculated exactly via

$$
\begin{aligned}
\left(M_{\nu}\right)_{\alpha \beta}= & \sum_{i} \frac{h_{\alpha i} h_{\beta i} M_{N i}}{16 \pi^{2}} \\
& \times\left[\sum_{j}\left(U_{R}\right)_{1 j}\left[\frac{m_{R j}^{2}}{m_{R j}^{2}-M_{N i}^{2}} \ln \left(\frac{m_{R j}^{2}}{M_{N i}^{2}}\right)\right]\right. \\
& \left.-\sum_{j}\left(U_{I}\right)_{1 j}\left[\frac{m_{I j}^{2}}{m_{I j}^{2}-M_{N i}^{2}} \ln \left(\frac{m_{I j}^{2}}{M_{N i}^{2}}\right)\right]\right]
\end{aligned}
$$

where $U_{R}\left(U_{I}\right)$ is the unitary matrix that makes $m_{R}\left(m_{I}\right)$ mass eigenstates and $h_{\alpha i}$ is the parameter for the interactions $\nu N \eta_{L 2}^{0}$ and $\nu \tilde{N} \eta_{L 2}{ }^{0}$.

Both diagrams require supersymmetry breaking to be nonzero. The one on the right needs the $A$ term $\phi_{L 1}^{0} \eta_{L 2}^{0} \tilde{\zeta}_{3}$ twice and the $B$ term $\tilde{\zeta}_{3} \tilde{\zeta}_{3}$ once whereas the one on the left requires only the $B$ term $\tilde{N} \tilde{N}$ once. We expect thus the latter diagram to be much more important. We estimate its contribution to be

$m_{\nu} \simeq \frac{h^{2} v_{L 1}^{2} \Delta M_{N}^{2}}{16 \pi^{2} M_{N}^{3}}$, 
Fig. 1 Scotogenic neutrino mass
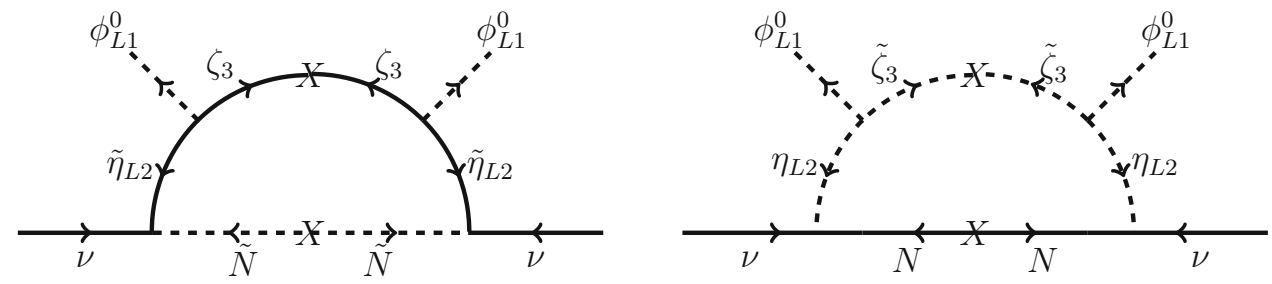

where $h$ is the diagonal Yukawa coupling, $\Delta M_{N}^{2}$ is the supersymmetry breaking $B$ term, and $M_{3} \simeq M_{N}$ has been assumed. Using $v_{L 1} \simeq 100 \mathrm{GeV}, \Delta M_{N}^{2} \simeq 1 \mathrm{TeV}^{2}$, and $M_{N} \simeq 10^{5} \mathrm{GeV}$, and $h^{2} \simeq 10^{-3}$, we find $m_{v} \simeq 0.1 \mathrm{eV}$.

The issue of radiative neutrino mass through dark matter, with or without supersymmetry, has been thoroughly discussed in previous papers [1-11]. Here we concentrate on the interplay between the possible dark-matter candidates due to this mechanism with those of the other two mechanisms, i.e. supersymmetry and left-right symmetry, as described below.

\section{Dark-matter candidates of the model}

There are three conserved quantities in this model: a global $U$ (1) number $S^{\prime}=S+T_{3 R}$, with $S^{\prime}=0$ for the usual quarks and leptons and $S^{\prime}=1$ for the scotino $n$, and the discrete $Z_{2}$ symmetries $M$ and $H$. The usual $R$ parity is then $R \equiv M H(-1)^{2 j}$. The various superfields of this model under $S^{\prime}, M$, and $H$ are listed in Table 2.

The existence of two $Z_{2}$ symmetries ( $M$ and $H$ ) allows for four different combinations $(++,+-,-+,--)$, with $(+)$ being even and ( - ) being odd under the respective $Z_{2}$ parities to have stable particles. A quick examination shows that the only particle that has $(-,-)$ is the Majorana fermion $N$,

Table 2 Superfields under $S^{\prime}=S+T_{3 R}, M$, and $H$

\begin{tabular}{llll}
\hline$S^{\prime}$ & $M$ & $H$ & Superfields \\
\hline 0 & - & + & $u, d, v, e$ \\
0 & + & + & $g, \gamma, W_{L}^{ \pm}, Z, Z^{\prime}$ \\
0 & + & + & $\phi_{L 1}^{0}, \phi_{L 1}^{-}, \phi_{L 2}^{+}, \phi_{L 2}^{0}, \phi_{R 1}^{0}, \phi_{R 2}^{0}$ \\
0 & + & + & $\delta_{11}^{0}, \delta_{11}^{-}, \delta_{22}^{+}, \delta_{22}^{0}$ \\
1 & - & + & $n, h^{c}$ \\
-1 & - & + & $n^{c}, h$ \\
1 & + & + & $W_{R}^{+}, \phi_{R 2}^{+}, \delta_{12}^{+}, \delta_{12}^{0}$ \\
-1 & + & + & $W_{R}^{-}, \phi_{R 1}^{-}, \delta_{21}^{0}, \delta_{21}^{-}$ \\
0 & - & - & $N$ \\
0 & + & - & $\eta_{L 1}^{0}, \eta_{L 1}^{-}, \eta_{L 2}^{+}, \eta_{L 2}^{0}, \eta_{R 1}^{-}, \eta_{R 2}^{+}$ \\
0 & + & - & $\zeta_{1}^{-}, \zeta_{2}^{+}, \zeta_{3}$ \\
1 & + & - & $\eta_{R 1}^{0}$ \\
-1 & + & - & $\eta_{R 2}^{0}$ \\
\hline
\end{tabular}

as noted in Table 2. In Sect. 3, it was seen that $N$ contributes in loop (see Fig. 1 for example) to yield neutrino masses and hence it must be heavy ( $\sim 100 \mathrm{TeV})$ to account for the smallness of the neutrino masses. This makes $N$ too heavy to be a DM candidate. For $(-+)$, the only possible DM candidate is the scotino $n$. Note that a scalar particle (such as the sneutrino or the scalar partner of the scotino with $(-+))$ is excluded from being a DM candidate given its large coupling with the $Z$ boson and a large direct-detection cross section. With the combination $(++)$, the only possibility is to have the lightest neutralino eigenstate. Finally, $(+-)$ contains exotic scalars and their superpartners (Higgsinos), which, in principle, can be DMs. However, one can show (for example) that for the scalar $\eta_{R}$, the annihilation cross section is proportional to the velocity $\langle\sigma v\rangle \propto v$, and hence it is not a good DM candidate. Also the singlet $\zeta_{3}$ (which is in a mixed state with $\eta_{L}$ ) has a large mass, $\sim 50 \mathrm{TeV}$, given the gauge-coupling unification. The singlet will not be a candidate, but the decoupled $\eta_{L}$ will be.

Hence, a possible scenario for dark matter is to have the following three coexisting stable particles [18]: the lightest neutralino $\tilde{\chi}_{1}^{0}\left(S^{\prime}=0, H=+, R=-\right)$, the lightest scotino $n\left(S^{\prime}=1, H=+, R=+\right)$, and the exotic $\tilde{\eta}_{R}^{0}$ fermion ( $S^{\prime}=1, H=-, R=+$ ). One should note here that the $\tilde{\eta}_{R}^{0}$ fermion is a type of neutralino, but it does not mix with gauginos and other Higgsinos and that is how it differs from the lightest neutralino $\tilde{\chi}_{1}^{0}$ of this model, making it possible to be much heavier than the LSP and still be stable. The dark sector may be far from just the one particle that is usually assumed, as in the MSSM. In the presence of several darkmatter candidates, the one with the largest annihilation cross section contributes the least, but it may be the first to be discovered at the large hadron collider (LHC). This means that in this model, the severe constraint due to dark-matter relic abundance on the one candidate particle of the MSSM, i.e. the lightest neutralino, may be relaxed, because it needs only to account for a fraction of the total dark-matter abundance. The allowed parameter space of the MSSM becomes much bigger and the opportunity for its discovery is enhanced at the LHC.

In Fig. 2, we show the gauge-coupling unification of this model [16]. The $U(1)_{Y}$ coupling runs until the $S U(2)_{R}$ breaking scale $M_{R}$, where $\alpha_{X}^{-1}\left(M_{R}\right)=\alpha_{Y}^{-1}\left(M_{R}\right)+$ $\alpha_{L}^{-1}\left(M_{R}\right)$ and $\alpha_{L}=\alpha_{R}$. After $M_{R}$, the gauge symmetry 
$S U(2)_{L}$ and $S U(2)_{R}$ are unified with the coupling $\alpha_{L R}$. The unified gauge group is $S O(10)$. From the requirement of gauge-coupling unification, it was shown that if the $S U(2)_{R}$ breaking scale $M_{R}$ equals the supersymmetry breaking scale $M_{S}$, the mass scale $M_{X}$ of the singlet superfields $\zeta_{1,2,3}$ should obey

$M_{R}^{7 / 4} M_{X}^{-3 / 4} \simeq 53.28 \mathrm{GeV}$

Given that the LHC has not seen any evidence of supersymmetry up to now, we can set $M_{R} \geq 1 \mathrm{TeV}$. In that case, $M_{X} \geq 50 \mathrm{TeV}$ (the dashed line in Fig. 2 is included to easily observe the change of slope at $M_{X}$ in the running of $\alpha_{X}$ ). As a result, interactions involving $\zeta_{1,2,3}$ may be ignored in our studies of dark matter. We further assume that the $N_{1,2,3}$ singlets are also heavy, so they may also be ignored.

For our scenario, we assume the masses $m_{\chi}, m_{n}, m_{\eta}$ of the three stable dark-matter particles $\tilde{\chi}_{1}^{0}, n, \tilde{\eta}_{R}^{0}$ to be arranged in ascending order. $\tilde{\eta}_{R}^{0}$ has $I_{3 L}=0$, so it couples only to $Z^{\prime}$. Hence the annihilation of $\tilde{\eta}_{R}^{0} \overline{\tilde{\eta}}_{R}^{0}$ to $Z^{\prime}$ to particles with

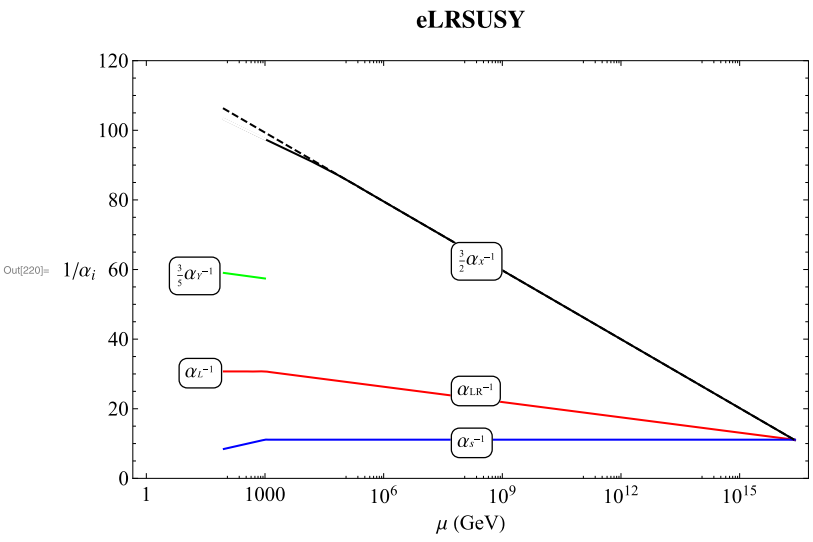

Fig. 2 Gauge-coupling unification in this model

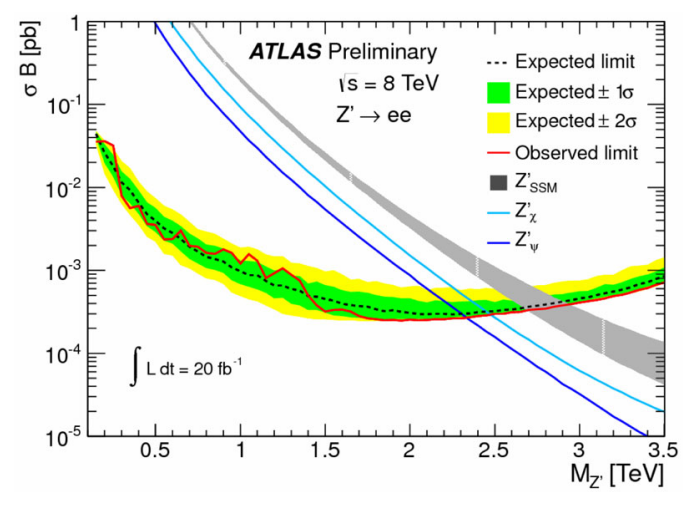

masses smaller than $m_{\eta}$ will determine its relic abundance. Once $\tilde{\eta}_{R}^{0}$ freezes out, we need to consider the interactions of $n$. Again $n$ has $I_{3 L}=0$, so it couples to $Z^{\prime}$, but there is also the interaction $\bar{e} n^{c} W_{R}^{-}$. Hence the annihilation of $n \bar{n}$ occurs through $Z^{\prime}$ to particles with mass smaller than $m_{n}$ as well as to $e^{+} e^{-}$through $W_{R}^{ \pm}$exchange. This will determine the relic abundance of $n$. After $n$ freezes out, the remaining particles are presumably those of the MSSM, and the annihilation of $\tilde{\chi}_{1}^{0} \tilde{\chi}_{1}^{0}$ will determine its relic abundance.

This added flexibility should relax some of the most stringent constraints facing the MSSM today.

\subsection{Bound on $Z^{\prime}$ from LHC data}

$Z^{\prime}$ couples to the current $[12,15]$

$J_{Z^{\prime}}=s_{R}^{2} J_{3 L}+c_{L}^{2} J_{3 R}-s_{R}^{2} J_{e m}$,

with strength $g_{Z^{\prime}}=e / s_{R} c_{L} \sqrt{c_{L}^{2}-s_{R}^{2}}$. Given the unification requirements in [16], we assume $g_{L}=g_{R}$, which implies $\sin \theta_{R}=\sin \theta_{L} \equiv \sin \theta_{W}$. We evaluate the bound on the mass of $Z^{\prime}$ in our model from LHC data with $E_{C M}=8 \mathrm{TeV}$ and integrated luminosity of $20 \mathrm{fb}^{-1}$. The result is shown in Fig. 3. On the left, we show the figure from ATLAS [19], where the bound was obtained for producing $Z^{\prime}$ and subsequent decays to $e^{ \pm} e^{\mp}$ for some popular $Z^{\prime}$ models. The right hand side shows our model cross sections in blue and the bound from LHC data in red, as seen in the LHS of the figure. The cuts on the electron $p_{T}>40 \mathrm{GeV}$ and pseudorapidity $|\eta|<2.47$ have been employed to obtain the signal in our model. We use the event generator CalcHEP [20] for calculating the cross section and use the CTEQ6L parton distribution function [21]. From Fig. 3 we obtain the bound on the mass of $Z^{\prime}, M_{Z^{\prime}}=2.045 \mathrm{TeV} \simeq 2 \mathrm{TeV}$. The bound on SSM, the phenomenological $Z^{\prime}$ model with SM coupling, has been cross-checked to be around $2.8 \mathrm{TeV}$, as shown on the left hand side.

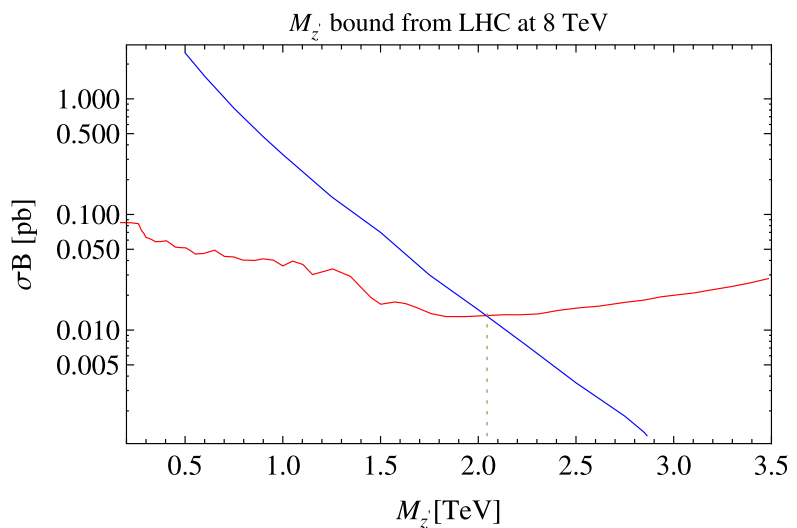

Fig. 3 LHS Bound on different $Z^{\prime}$ masses at LHC from ATLAS with $E_{C M}=8 \mathrm{TeV}$ and integrated luminosity of $20 f b^{-1}$. $R H S$ The limit is exploited to determine the bound on the $Z^{\prime}$ mass of this model 


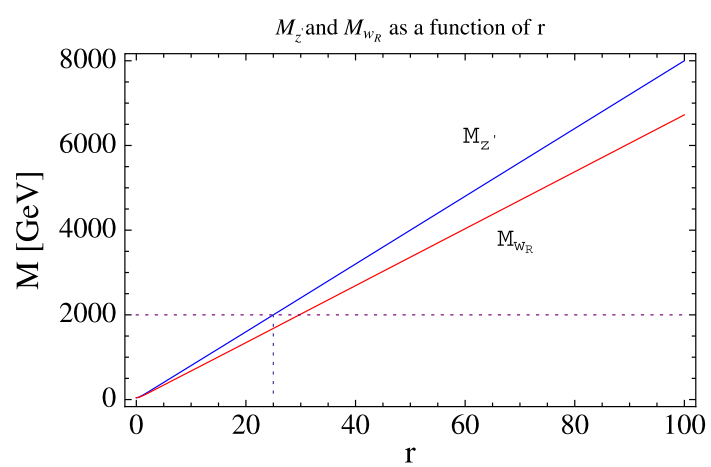

Fig. 4 Linear dependence of $M_{Z^{\prime}}$ (blue) and $M_{W_{R}}$ (red) on the ratio of Higgs vevs $r$ as defined in Eq. 7. A horizontal dotted line indicates the bound from $\mathrm{LHC}$ on the $Z^{\prime}$ mass at $2 \mathrm{TeV}$

The gauge boson masses are calculated to be

$$
\begin{aligned}
M_{Z} & =\frac{g_{L} v_{L}}{\sqrt{2\left(1-2 \sin ^{2} \theta_{W}\right)}}, \\
M_{Z^{\prime}} & =M_{Z} \sqrt{\sin ^{2} \theta_{W}+r^{2} \cos ^{2} \theta_{W}}, \\
M_{W_{R}} & =M_{Z} \sqrt{\sin ^{2} \theta_{W}+r^{2}\left(\cos ^{2} \theta_{W}-\sin ^{2} \theta_{W}\right)} .
\end{aligned}
$$

Here $\left(v_{L}^{2} / u^{2}\right)=\left(1-2 \sin ^{2} \theta_{W}\right) / \sin ^{2} \theta_{W}$ has been used to assume zero $Z-Z^{\prime}$ mixing, and we have defined the ratio of the Higgs vacuum expectation values as

$r=\frac{v_{R}}{v_{L}}$

with $v_{L}^{2}=v_{L 1}^{2}+v_{L 2}^{2}, v_{R}^{2}=v_{R 1}^{2}+v_{R 2}^{2}$ and $u^{2}=u_{1}^{2}+u_{4}^{2}$.

In Fig. 4, we show the linear dependence of the $Z^{\prime}$ and $W_{R}$ mass on the ratio of Higgs vacuum expectation values $r$ following Eq. 6. We note that the mass of $Z^{\prime}$ is larger than $W_{R}$ for $M_{Z}^{\prime} \geq 30 \mathrm{GeV}$. The bound on $M_{Z}^{\prime} \geq 2 \mathrm{TeV}$ from LHC eventually put a bound of $r \geq 25$, as shown. In the following analysis, we use $r$ as a plotting variable instead of $M_{Z}^{\prime}$ or $M_{W_{R}}$.

\subsection{Relic abundance of $n$ and $\tilde{\eta}_{R}^{0}$}

The annihilation cross sections for DM $\tilde{\eta}_{R}^{0}$ to SM particles goes through s-channel diagram exchanging $Z^{\prime}$, while $n$ has an additional piece through a t-channel diagram to $e_{R}^{ \pm}$through $W_{R}^{ \pm}$exchange. The Feynman diagrams are shown in Figs. 5 and 6.

The expressions for thermally averaged cross section $(\langle\sigma v\rangle)$ for these two DM components meaning annihilation in SM are indicated in Eqs. 8 and 9. We have

$$
\langle\sigma v\rangle_{\eta} \simeq \frac{g_{L}^{4}}{64 \pi} \frac{m_{\tilde{\eta}_{R}^{0}}^{2}}{\left(4 m_{\tilde{\eta}_{R}^{0}}^{2}-M_{Z^{\prime}}^{2}\right)^{2}} \frac{\left(10-29 c_{W}^{2}+22 c_{W}^{4}\right)}{\left(2 c_{W}^{2}-1\right)^{2}},
$$

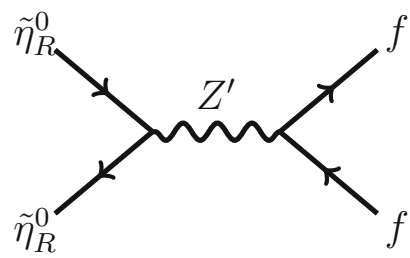

Fig. 5 Feynman diagram for $\tilde{\eta}_{R}^{0}$ annihilation to SM
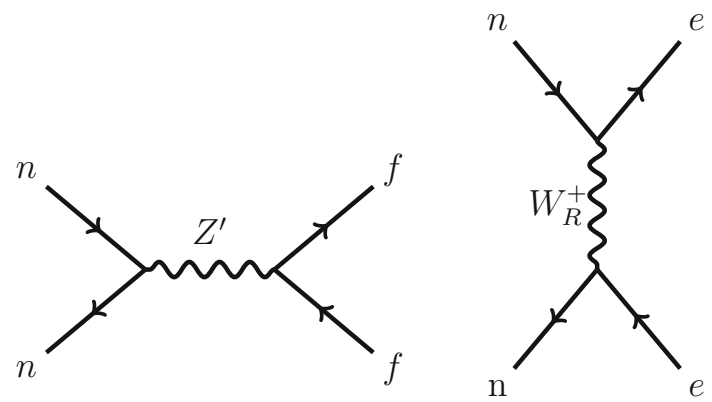

Fig. 6 Feynman diagram for $n$ annihilation to SM

$$
\begin{aligned}
& \langle\sigma v\rangle_{n} \simeq \frac{g_{R}^{4} m_{n}^{2}}{64 \pi}\left[\frac{10 s_{W}^{4}-9 s_{W}^{2} c_{W}^{2}+3 c_{W}^{4}}{\left(4 m_{n}^{2}-M_{Z^{\prime}}^{2}\right)^{2}\left(c_{W}^{2}-s_{W}^{2}\right)^{2}}\right. \\
& \left.+\frac{3}{\left(m_{n}^{2}+M_{W_{R}}^{2}\right)^{2}}+\frac{3\left(c_{W}^{2}-2 s_{W}^{2}\right)}{\left(4 m_{n}^{2}-M_{Z^{\prime}}^{2}\right)\left(m_{n}^{2}+M_{W_{R}}^{2}\right)\left(c_{W}^{2}-s_{W}^{2}\right)}\right] .
\end{aligned}
$$

With the unification condition, $g_{R}^{2}=g_{L}^{2} \simeq 0.427$ and $\sin ^{2} \theta_{W}=0.23$, numerically we obtain

$$
\begin{aligned}
\langle\sigma v\rangle_{\eta} \simeq & \frac{0.00222 m_{\eta_{R}^{0}}^{2}}{4 m_{\tilde{\eta}_{R}^{0}}{ }^{2}-M_{Z}^{\prime 2}}, \\
\langle\sigma v\rangle_{n} \simeq & \frac{0.00222 m_{n}{ }^{2}}{4 m_{n}{ }^{2}-M_{Z}^{\prime 2}}+\frac{0.00272 m_{n}{ }^{2}}{m_{n}{ }^{2}+M_{W_{R}}{ }^{2}} \\
& +\frac{0.00156 m_{n}{ }^{2}}{\left(4 m_{n}{ }^{2}-M_{Z}^{\prime 2}\right)\left(m_{n}{ }^{2}+M_{W_{R}}{ }^{2}\right)} .
\end{aligned}
$$

If we assume that the decouplings of $\tilde{\eta}_{R}^{0}, n$, and $\tilde{\chi}_{1}^{0}$ from the hot soup of SM particles are independent of the interactions with each other, the relic density for each DM component can be approximated by

$\Omega_{i} h^{2} \simeq \frac{0.1 p b}{\langle\sigma v\rangle_{i}}$.

The total abundance will be the sum of the three DM components, i.e.

$\Omega_{\mathrm{DM}_{\mathrm{tot}}} h^{2}=\Omega_{\eta} h^{2}+\Omega_{n} h^{2}+\Omega_{\chi_{1}^{0}} h^{2}$. 

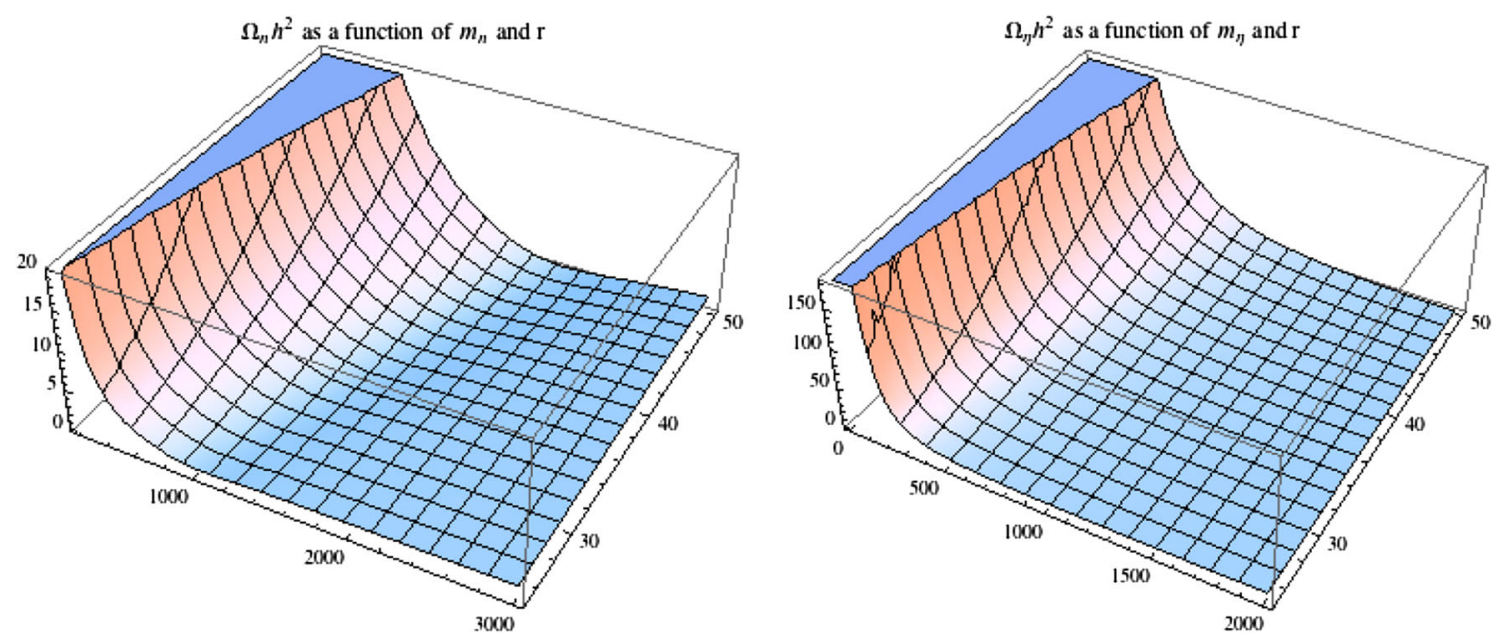

Fig. 7 A 3-dimensional plot showing $\Omega h^{2}$ (z-axis) dependence on mass ( $x$-axis) and $r$ (y-axis). LHS $n$ and $R H S \tilde{\eta}_{R}^{0}$

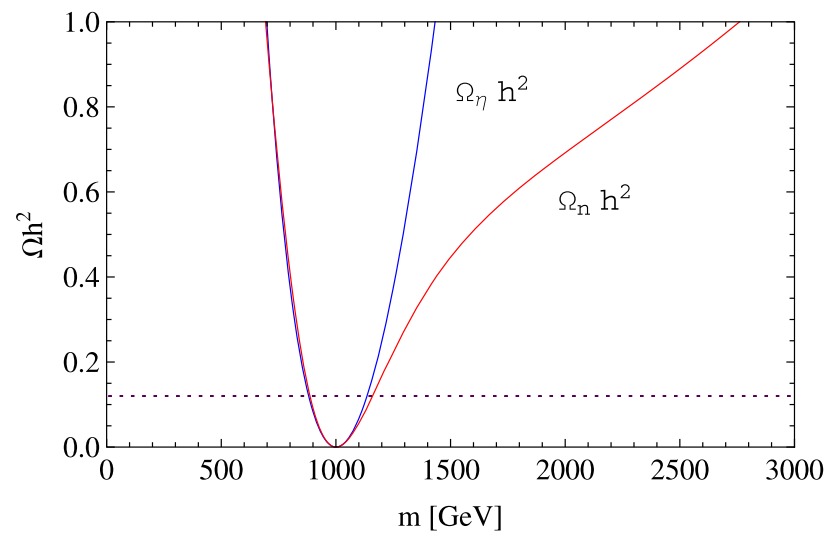

Fig. $8 \Omega h^{2}$ dependence for DM $n$ and $\tilde{\eta}_{R}^{0}$ on mass for $r=25$

With this assumption, we evaluate the relic abundance for each of the DM components and look for the parameter space where they add up to the constraint from WMAP [22]. ${ }^{1} \mathrm{We}$ have

$0.094<\Omega_{\mathrm{DM}_{\mathrm{tot}}} h^{2}<0.130$.

In Fig. 7, we show a 3-dimensional plot with $\Omega h^{2}$ along the $\mathrm{z}$-axis, DM mass $m$ along the $\mathrm{x}$-axis and the ratio of Higgs vevs $r$ along the y-axis for the DM component $n$ on LHS and $\tilde{\eta}_{R}^{0}$ on RHS. We use Eqs. 10, 11, and 12 to draw them. Both of the DMs show similar behavior. Now, a cut along the $r$-axis at 25, shows the dependence of $\Omega h^{2}$ on DM mass $m$ which is shown in Fig. 8. The difference in $n$ and $\tilde{\eta}_{R}^{0}$ annihilation is clear from this.

In the three component DM framework, we study a scenario where the two components $n$ and $\tilde{\eta}_{R}^{0}$ dominate in the

${ }^{1}$ PLANCK [23] data essentially indicates a very similar range, though more stringent, almost indistinguishable from WMAP in the present context. relic abundance leaving a very tiny space for the neutralino $\tilde{\chi}_{1}^{0}$. We will discuss neutralino DM shortly. For example, we focus on the region of parameter space, where

$\Omega_{\eta} h^{2}+\Omega_{n} h^{2}=0.1$

In such a case, if we assume in addition that each of the components contribute equally, then we end up with Fig. 9. This indicates that we obtain two possible masses for a given value of $r$ and $\Omega h^{2}$ and the difference in $n$ and $\tilde{\eta}_{R}^{0}$ annihilation does not matter in the range of $r$ and $\Omega h^{2}$ we are interested in. This is shown in the top panel of Fig. 9, for $n$ (left) and $\tilde{\eta}_{R}^{0}$ (right). They look exactly the same, where DM mass is plotted with $r$. In the bottom panel, we show the case when one of the components contributes fully to the relic abundance with $\Omega_{i} h^{2}=0.1$.

Equation 15 is appropriately depicted in Fig. 10 for different $Z^{\prime}$ masses. They are represented by three circles (the circular shape is understandable from looking at Fig. 8) in the $m_{n}$ and $m_{\tilde{\eta}_{R}^{0}}(\mathrm{GeV})$ plane for $M_{Z}^{\prime}=2,3$ and $4 \mathrm{TeV}$ around $m_{n}=m_{\tilde{\eta}_{R}^{0}}=M_{Z}^{\prime} / 2$. The reason is simple; the resonance region essentially contributes for relic abundance. We highlight the case for $M_{Z}^{\prime}=2 \mathrm{TeV}$ in the RHS of Fig. 10. The whole region in green becomes allowed when we have the condition $\Omega_{\eta} h^{2}+\Omega_{n} h^{2} \leq$ 0.12 (i.e. the contour shrinks for a smaller abundance). We also note that, if we adhere to the assumption made initially that $m_{\eta} \geq m_{n}$, then only half of the circle above the diagonal line is allowed for relic abundance restricting the allowed mass range for $n$ between $866-1100 \mathrm{GeV}$ and for $\tilde{\eta}_{R}^{0}$ between $915-1163 \mathrm{GeV}$. Given that the plot is close to a perfect circle, $\Omega_{n} h^{2} \geq \Omega_{\eta} h^{2}$ in this limit. Hence, if $n$ and $\tilde{\eta}_{R}^{0}$ together contribute $90 \%$ of the total dark matter relic density, $\tilde{\eta}_{R}^{0}$ can contribute $1-45 \%$ and $n$ can contribute $45-90 \%$. 

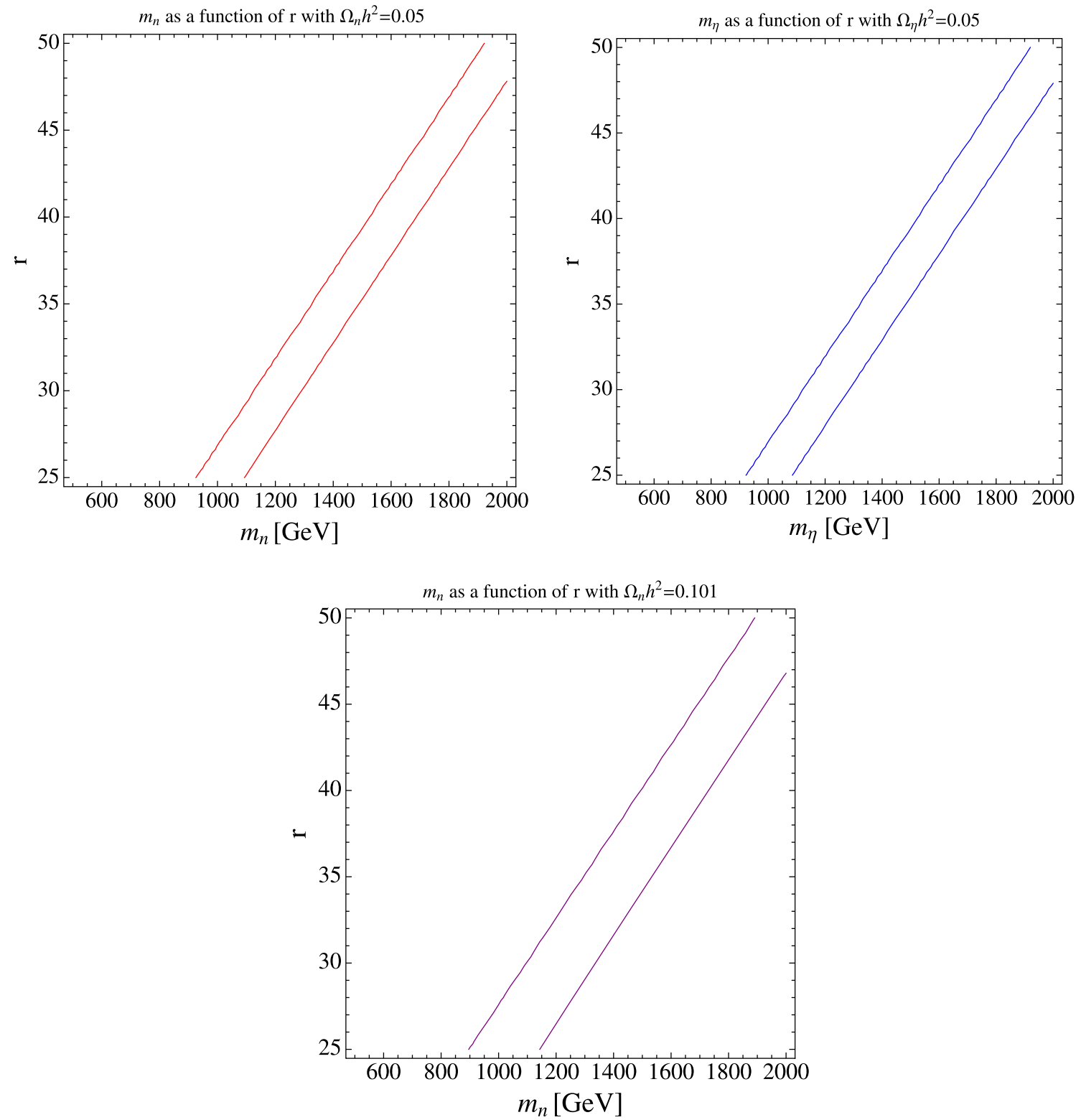

Fig. 9 Top $m-r$ dependence when each of the DM components $n$ (left) and $\tilde{\eta}_{R}^{0}(r i g h t)$ contributes equally to the relic density with $\Omega_{i} h^{2}=0.05$. Bottom When one component dominates, i.e. $\Omega_{i} h^{2}=0.1$. Masses are in $\mathrm{GeVs}$

\subsection{Direct detection of $n$ and $\tilde{\eta}_{R}^{0}$}

Direct detection of $n$ and $\tilde{\eta}_{R}^{0}$ takes place through the t-channel $Z^{\prime}$ interaction with quarks. The Feynman graph is shown in Fig. 11. Due to only this contribution, the spin-independent (SI) cross section is very small.

We use MicrOMEGAs [24] to calculate the effective SI nucleon scattering cross section. The parton-level interaction is converted to the nucleon level by using effective nucleon $f_{q}{ }^{N}(N=p, n)$ couplings defined as [24]

$\left\langle N\left|m_{q} \bar{\psi}_{q} \psi_{q}\right| N\right\rangle=f_{q}{ }^{N} M_{N}$, where $M_{N}$ is the nucleon mass and we use the default form factors in [24] as $f_{u}^{p}=0.033, f_{d}^{p}=0.023, f_{s}^{p}=0.26$, for the proton; $f_{u}^{n}=0.042, f_{d}^{n}=0.018, f_{s}^{n}=0.26$ for the neutron; while for the heavy quarks the $f_{q}^{N}$ are generated by gluon exchange with the nucleon and are given by

$f_{Q}^{N}=\frac{2}{27}\left(1-\sum_{q=u, d, s} f_{q}^{N}\right) \quad Q=c, t, b$.

The results are shown in Fig. 12. The bounds from XENON100 (above) and XENON1T (below) are shown by two continuous lines in purple and red, respectively. Any 
Fig. 10 LHS A plot showing $m_{n}-m_{\eta}(\mathrm{GeV})$ contours for $\Omega_{n} h^{2}+\Omega_{\eta} h^{2}=0.1$ for $M_{Z}^{\prime}=$ 2, 3, 4 TeV. RHS Region of the $m_{n}-m_{\eta}(\mathrm{GeV})$ parameter space when $\Omega_{n} h^{2}+\Omega_{\eta} h^{2} \leq 0.12$ with $M_{Z}^{\prime}=2 \mathrm{TeV}$
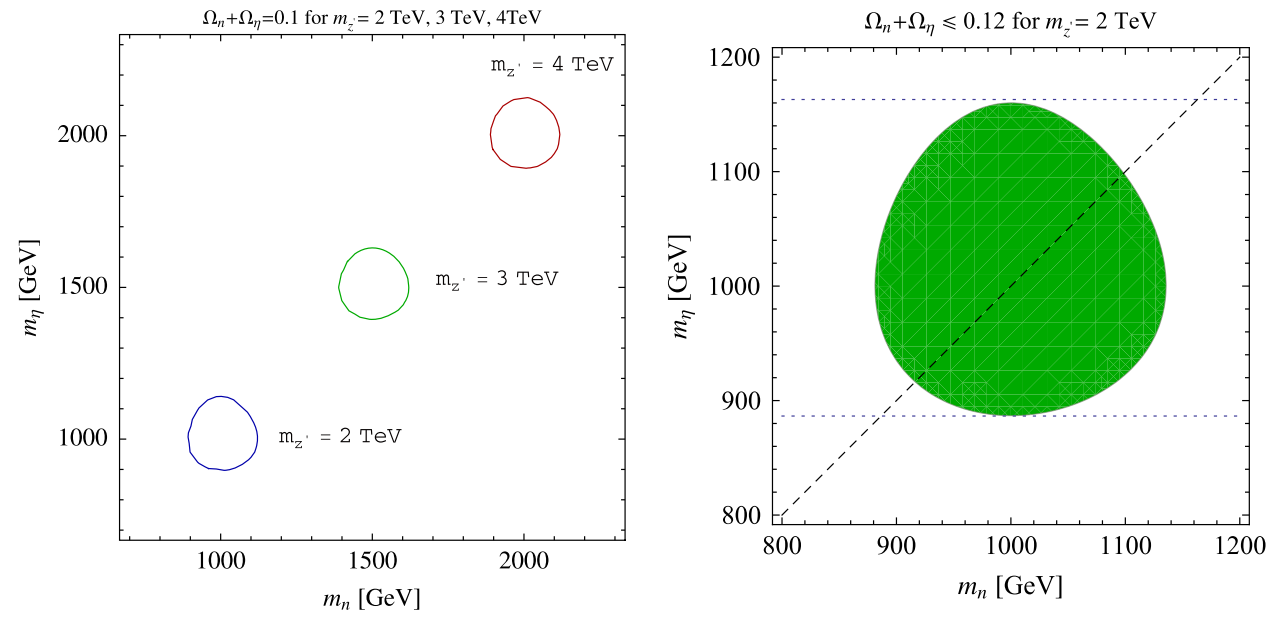

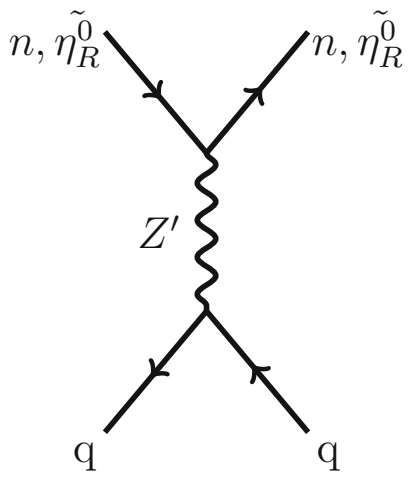

Fig. 11 Diagram for scattering with quarks for direct detection

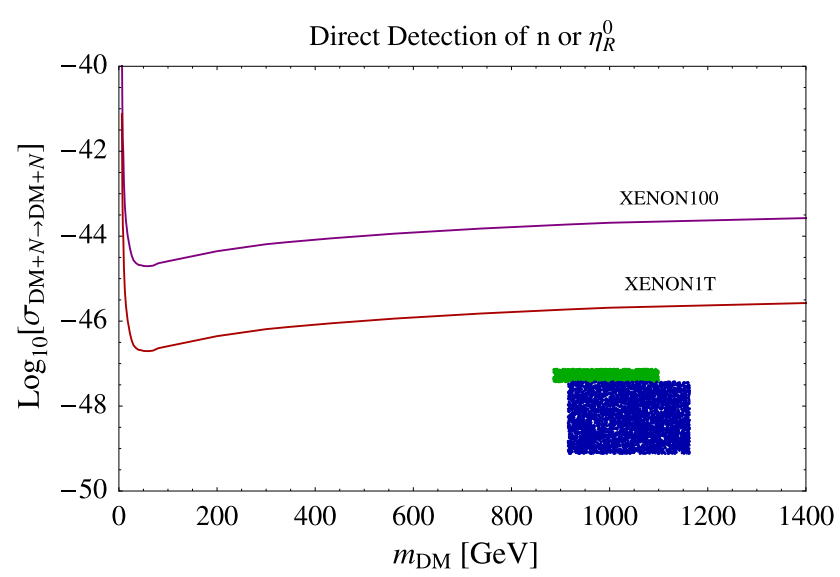

Fig. 12 Direct-detection constraint for DM $n$ and $\tilde{\eta}_{R}^{0}$. The spinindependent effective nucleon cross section $\left(\mathrm{cm}^{2}\right)$ in $\log$ scale is plotted along the $y$-axis as a function of DM mass $(\mathrm{GeV})$ along the $x$-axis. The upper thick curve in purple shows the limit from XENON100 and the lower one in red is for XENON1T. Points in the blue box represent $\tilde{\eta}_{R}^{0}$ contributing 1-45\% (bottom to top) and those in green correspond to $n$ contributing $45-90 \%$ (bottom to top) of the total dark-matter density in the WMAP allowed mass range

points above the XENON100 lines will be discarded by the direct search experiments. In Fig. 12, points in blue show the results of SI direct-detection cross section for $\tilde{\eta}_{R}^{0}$ with $M_{Z}^{\prime}=$
$2 \mathrm{TeV}$ and those in green represent $n$ within the allowed mass range to obtain the correct relic density; $m_{n}$ is between 866 $1100 \mathrm{GeV}$ and $m_{\eta}$ between 915-1163 GeV. Although $n$ and $\tilde{\eta}_{R}^{0}$ have the same quark interaction as in Fig. 11 and have the same direct-detection cross section, given the mass hierarchy $m_{\eta} \geq m_{n}, n$ contributes more than $\tilde{\eta}_{R}^{0}$ to the dark-matter density. Due to the multi-component nature of the dark matter, the effective direct-detection cross section for each DM component is obtained by multiplying the fraction of their number density $\frac{n_{\mathrm{DM}}}{n_{\text {tot }}}$ with the actual nucleon cross section $\sigma_{N}$ (assuming that all of the DMs are accessible to the detector). We have

$\sigma_{N \text { eff }}=\frac{n_{\mathrm{DM}}}{n_{\mathrm{tot}}} \sigma_{N} \simeq \frac{\Omega_{\mathrm{DM}} h^{2}}{\Omega_{\mathrm{tot}} h^{2}} \sigma_{N}$

The thickness of the direct-detection cross section essentially comes from the fraction $\frac{n_{\mathrm{DM}}}{n_{\text {tot }}}$, which has been varied between 1-45\% for $\tilde{\eta}_{R}^{0}$ (in blue) and 45-90\% for $n$ (in green). Hence, points at the bottom of the blue box constitute only $1 \%$, while those at the top in green constitute $90 \%$ of the total DM. The unequal thickness in the blue and green boxes is due to the logarithmic scale of the effective cross section.

The direct-detection cross section also does not depend on the DM mass, while it very heavily depends on the $Z^{\prime}$ mass. With higher $M_{Z}^{\prime}$ they go down even below this value, making it harder to perform a direct search. The possibility of early discovery of these DMs in near-future experiments seems to be small, although they are surely allowed by the exclusion limits set by XENON.

\subsection{Relic abundance and direct detection of wino type of neutralino $\tilde{\chi}_{1}^{0}$}

Let us now discuss the lightest neutralino $\left(\tilde{\chi}_{1}^{0}\right)$ as the third DM candidates in this three component DM set up. The 
Neutralino Composition \& Masses

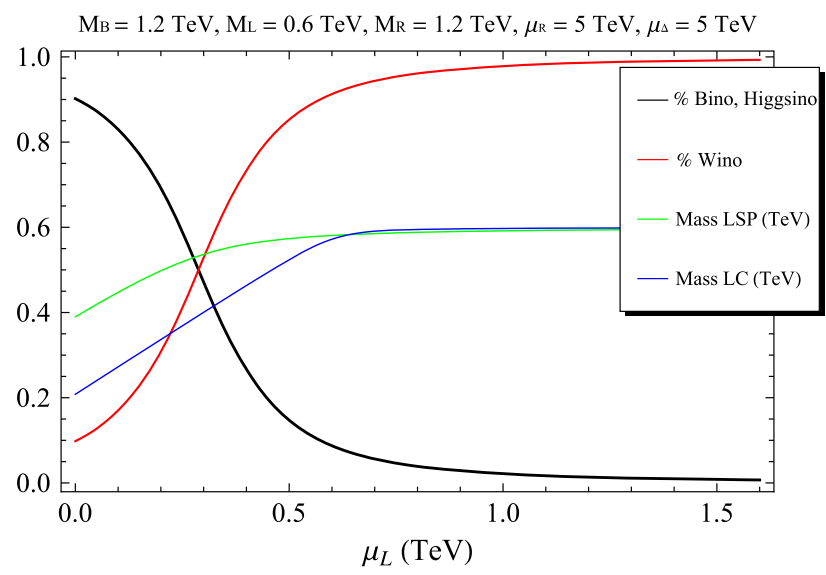

Fig. 13 Plot showing a limit when the lightest neutralino becomes predominantly a wino and the first chargino becomes degenerate with LSP

neutralino sector in this extended LR SUSY model is nontrivial and is constituted of three gauginos $\left(M_{B}, M_{L}, M_{R}\right)$ and 13 Higgsinos. Seven out of them, which are superpartners of the scalar fields that do not have a vev, do not mix with the gauginos or the rest of the Higgsinos. This yields a 9-dimensional neutralino mass matrix.

For simplicity, we take the limit where the neutralino DM is predominantly a wino. This is not a requirement of the model; in fact one could chose the lightest neutralino state to be a DM with admixtures from bino and Higgsino components. However, in the wino limit, the neutralino of this model can easily mimic the MSSM neutralino, with $M_{B}=$ $M_{1}, \mu_{L}=\mu, \beta_{L}=\beta$, and $1.43 M_{L}=M_{2}$. This is explicitly shown in the appendix. In Fig. 13, we show as an example that when $\mu_{L}$ (x-axis) is larger than $M_{L}$ (which we set at $0.6 \mathrm{TeV}$ ), the fraction of bino and Higgsino components in the lightest neutralino, in black thick line, goes almost to zero; giving rise to a wino DM with the red line reaching 1 . We also show that the lightest chargino (in blue, called LC) becomes degenerate with the lightest neutralino (in green) and both have masses around $600 \mathrm{GeV}$ in this particular point in parameter space. This degeneracy is a very well-known feature of the winodominated neutralino in MSSM. Note that in order to achieve this limit in this model, we kept $M_{R} \simeq M_{B}$, and the other non-MSSM parts were kept heavy, $\mu_{R}, \mu_{\Delta}=5 \mathrm{TeV}$.

It is also known that when the lightest chargino is degenerate with the neutralino DM, co-annihilation occurs [25], making the $\tilde{\chi}_{1}^{0}$ annihilation cross section much larger, yielding a very small abundance. This has been crafted in different ways [26-31] to make the wino a viable DM candidate by having moduli decay in anomaly-mediated SUSY breaking [27] or by non-thermal productions [31] etc. Wino DM has been studied also to justify PAMELA data [32,33]. However, the under-abundance works perfectly fine for us using the other two components for make up.

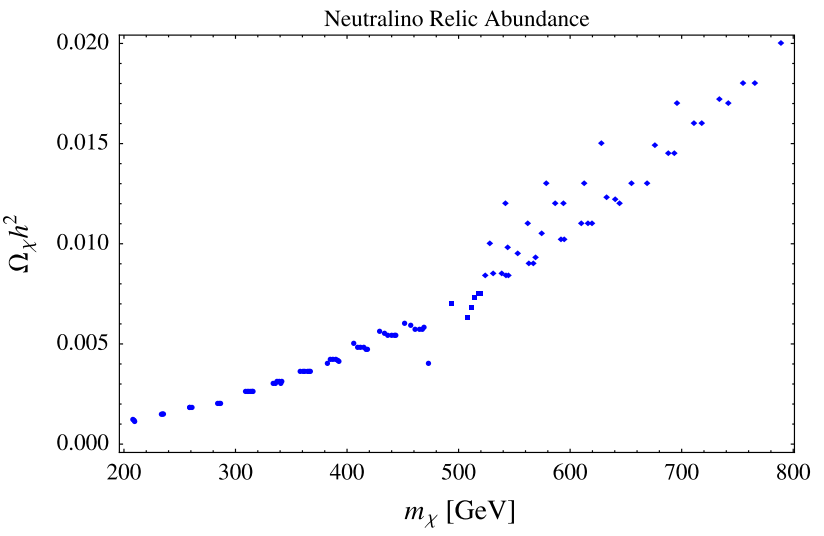

Fig. 14 Relic abundance of the lightest neutralino as a function of mass when it is predominantly a wino. The scanned parameter space range for $M_{1}$ is (800-1200) $\mathrm{GeV}$, for $M_{2}$ (200-775) GeV, and for $\mu$ (600-1000) GeV with $\mu, M_{1}>M_{2}$

Once again, we stress here that, while a bino DM is not possible given the overabundance it produces, the other regions of the neutralino DM parameter space, where it is an admixture of Higgsino-wino-bino and yields underabundance, is allowed for the model. Also this admixture is not only restricted to the MSSM-like part and includes non-MSSM Higgsinos; see the appendix for details. The lightest eigenvalue (and its interactions) can only be calculated numerically, essentially involving the whole parameter space of the model including the non-MSSM scalars introduced here. For simplicity, we only examine the region of the parameter space where the lightest neutralino is MSSM-like and predominantly a wino.

We show a sample scan of the wino-dominated neutralino for relic density and direct detection. The MSSM parameter space scanned here is as follows: $M_{1}$ between $800-1200 \mathrm{GeV}$, $M_{2} \simeq 1.43 M_{L}$, between $200-775 \mathrm{GeV}$, and the Higgsino parameter $\mu$ between 600-1000 GeV (with $\mu, M_{1}>M_{2}$ ). In Fig. 14, we show that the neutralino-DM under-abundance for $\Omega_{\chi_{1}^{0}} h^{2}$ is not larger than 0.02 if we keep $m_{\tilde{\chi}_{1}^{0}} \leq 800 \mathrm{GeV}$ (this follows from the assumption that the neutralino is the lightest of the three DMs and the limit can be increased for higher $Z^{\prime}$ mass). The neutralino DM constitutes only 1-20\% of the total DM density making Eq. 15 a good benchmark. Note that the scan yields predominantly a wino, but with some Higgsino component in it.

We use MicrOMEGAs [24] to evaluate the relic abundance and direct-detection cross sections for the neutralino DM which mimics MSSM in the parameter space mentioned above. The direct-detection cross section for the neutralino goes through t-channel processes as in Fig. 15. The squarks' contribution is negligible as they are heavy $\simeq 2 \mathrm{TeV}$. Also, for a pure wino, there is no Higgs channel and the $Z$-channel contributes more to the spin-dependent cross section. Hence, having some Higgsino fraction in the neutralino enhances 
Fig. 15 Diagram for the lightest neutralino $\tilde{\chi}_{1}^{0}$ scattering with quarks for direct detection

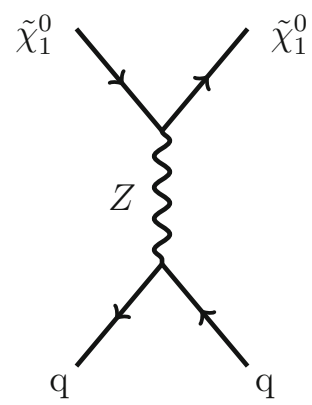

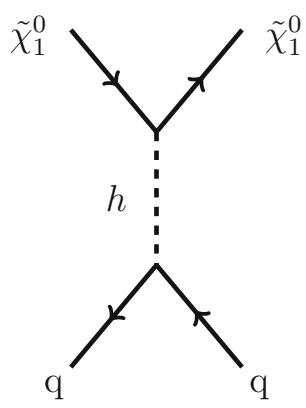

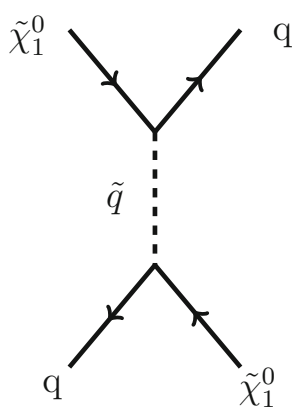

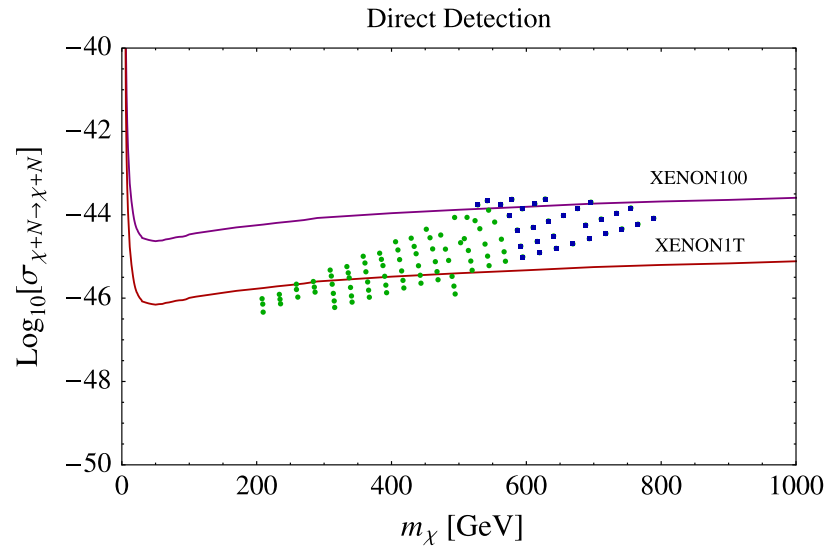

Fig. 16 Direct-detection constraint on the neutralino DM mass when it is predominantly wino. The upper curve is for XENON100 and the lower one is for XENON1T. Points in blue have a relic abundance higher than $10 \%$ and those in green have less. Scanned parameter space range for $M_{1}$ is $(800-1200) \mathrm{GeV}$, for $M_{2}(200-775) \mathrm{GeV}$, and for $\mu(600$ 1000) $\mathrm{GeV}$ with $\mu, M_{1}>M_{2}$

direct detection. In Fig. 16, we see that the neutralino can be accessible to direct-detection experiments in the near future with points close to XENON100 and XENON1T limit. Points in blue have a relic abundance contribution with more than $10 \%$ and they have an early detection possibility, while points in green have a relic density less than $10 \%$ and direct detection for them may be delayed depending on the mass and composition. While higher order calculations for direct detection of purely wino DM has been studied [34,35] and found to boost direct detection, we are not using them, since we are exploiting a small Higgsino fraction in the neutralino, which increases direct detection, while having co-annihilations to yield under-abundance.

It is worthwhile to clarify here that the accessibility of predominantly wino dark matter with a smaller Higgsino fraction to direct-detection experiments is quite achievable in MSSM itself with, for example, non-universal highscale gaugino masses in gravity mediated SUSY breaking; although it will not of course satisfy the relic abundance constraint. Here, we are choosing that suitable subspace of MSSM-like neutralino dark matter in our model which yields under-abundance to make up for the other two components and also is accessible to direct-detection experiments in the near future.

The mass range and the wino content in the neutralino studied here is consistent with the indirect-detection constraints from the Fermi gamma-ray space telescope or the high energy spectroscopic system (H.E.S.S.) [36].

We also note that the MSSM parameter space scan performed here does not correspond to a specific high-scale SUSY breaking pattern. So, the bounds on the chargino or neutralino masses obtained from LHC [37], which mostly assumes some specific high-scale pattern like minimal supergravity (mSUGRA) [38-42], are not applicable here.

It is also important to note that we did not study the entanglement of neutralino dark matter with the other components, only because we studied a particular region of the parameter space of neutralino DM, which contributes minimally to the relic abundance, while having possibilities of being detected in direct search experiments. However, in the most general case, one can study a similar entanglement of the neutralino DM with the other two components for relic abundance and direct detection.

In Fig. 17, we show a sample point in the three component dark-matter parameter space allowed by relic abundance with respect to the XENON100 and XENON1T direct-detection limit. In this point $\tilde{\chi}_{1}^{0}(644 \mathrm{GeV})$ in green, $n(912 \mathrm{GeV})$ in red, and $\tilde{\eta}_{R}^{0}(939 \mathrm{GeV})$ in blue constitute $10.7,62.5$, and $26.8 \%$ of the total DM density, respectively.

\section{Summary and outlook}

We have presented a consistent extension of the SM in which at least three stable dark-matter candidates coexist, as the result of three exactly conserved $Z_{2}$ symmetries. We have chosen as an example three specific dark-matter candidates: the lightest MSSM neutralino $\tilde{\chi}_{1}^{0}$, the lightest $S U(2)_{R}$ scotino $n$, and the exotic $\tilde{\eta}_{R}^{0}$ Higgsino, which generates a radiative neutrino mass. The three of them share in producing the correct dark-matter relic abundance of the Universe. We choose the MSSM $\tilde{\chi}_{1}^{0}$ to be dominantly the wino. This by itself would not be acceptable as a dark-matter candidate, because its usual co-annihilation with a chargino would result 


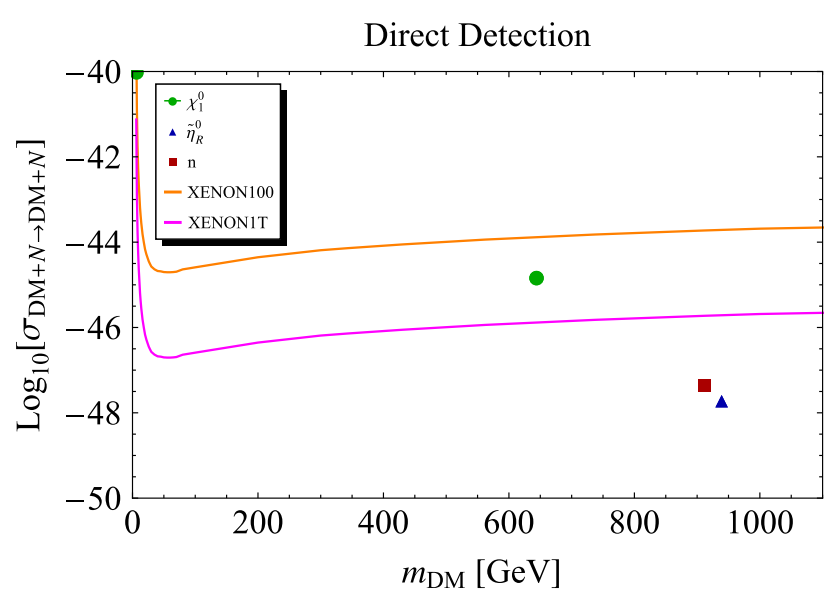

Fig. 17 A sample point in the three component dark-matter parameter space allowed by WMAP is plotted with respect to the XENON100 and XENON1T limits. $\tilde{\chi}_{1}^{0}(644 \mathrm{GeV})$ in green, $n(912 \mathrm{GeV})$ in red, and $\tilde{\eta}_{R}^{0}$ $(939 \mathrm{GeV})$ in blue constitute $10.7,62.5$, and $26.8 \%$ of the total DM density, respectively

in under-abundance. For our scenario, this is not a problem because we have two other dark-matter candidates to make up for the deficit. We choose the masses $m_{n}$ and $m_{\eta}$ to be around $1 \mathrm{TeV}$ so that they have a modest resonance enhancement in their annihilation to SM particles through the $Z^{\prime}$ of our model. From the LHC data, we find a lower bound of $2.045 \mathrm{TeV}$ on $M_{Z^{\prime}}$. With this value, the direct-detection cross section for $n$ and $\tilde{\eta}_{R}^{0}$ is calculated to lie between $10^{-47}-10^{-49}$ $\left(\mathrm{cm}^{2}\right)$ (depending on the fraction it contributes to the total DM density). This is at least an order of magnitude below the XENON1T detection limits. Nevertheless, a wino-dominated neutralino with a small Higgsino fraction is allowed by the relic abundance constraint in this multi-component setup and such $\tilde{\chi}_{1}^{0}$ does lie within the direct-detection limits.
It should be mentioned that we have not considered in detail the thermal history of the three assumed components of the dark matter. Neglected interactions between $n$ and $\tilde{\eta}_{R}^{0}$ can make the general situation more complicated and one needs to solve the coupled Boltzman equations involving $n, \tilde{\eta}_{R}^{0}$, and $\tilde{\chi}_{1}^{0}$ to study the exact decoupling of each DM component, depending on their relative masses and coupling strengths. This will be considered in future work.

The rich particle spectrum of this model in the $S U(2)_{R}$ sector makes it very likely to have interesting collider signatures at the LHC, with the production $Z^{\prime}$ as its most promising signal. These new particles may also open up new decay channels for sparticles thus altering the event rates in the lepton or jet-rich final states with missing energy. This difference from the MSSM may change the bounds on the sparticle masses at the LHC. We plan to elaborate on this in a future publication.

Acknowledgments This work is supported in part by the U.S. Department of Energy under Grant No. DE-FG03-94ER40837. The work of SB is supported by U.S Department of Energy under Grant No. DESC0008541. The work of DW is supported by UC MEXUS-CONACYT under Grant Reference No. 200406-303746.

Open Access This article is distributed under the terms of the Creative Commons Attribution License which permits any use, distribution, and reproduction in any medium, provided the original author(s) and the source are credited.

Funded by $\mathrm{SCOAP}^{3}$ / License Version CC BY 4.0.

\section{Appendix}

Using the basis

$\left(\tilde{\Psi}^{0}\right)^{T}=\left\{\tilde{B}, \tilde{W}_{L}, \tilde{\phi}_{L 1}, \tilde{\phi}_{L 2}, \tilde{W}_{R}, \tilde{\delta}_{11}, \tilde{\delta}_{22}, \tilde{\phi}_{R 1}, \tilde{\phi}_{R 2}\right\}$,

the neutralino mass matrix in our model is

$M_{\chi^{0}}=\left(\begin{array}{llll|lllll}M_{B} & 0 & -\frac{g_{1} v_{L 1}}{\sqrt{2}} & \frac{g_{1} v_{L 2}}{\sqrt{2}} & 0 & 0 & 0 & -\frac{g_{1} v_{R 1}}{\sqrt{2}} & \frac{g_{1} v_{R 2}}{\sqrt{2}} \\ 0 & M_{L} & \frac{g_{L} v_{L 1}}{\sqrt{2}} & -\frac{g_{L} v_{L 2}}{\sqrt{2}} & 0 & -\frac{g_{L} u_{1}}{\sqrt{2}} & \frac{g_{L} u_{4}}{\sqrt{2}} & 0 & 0 \\ -\frac{g_{1} v_{L 1}}{\sqrt{2}} & \frac{g_{L} v_{L 1}}{\sqrt{2}} & 0 & -\mu_{L} & 0 & 0 & \frac{f_{1} v_{R 2}}{2} & 0 & \frac{f_{1} u_{4}}{2} \\ \frac{g_{1} v_{L 2}}{\sqrt{2}} & -\frac{g_{L} v_{L 2}}{\sqrt{2}} & -\mu_{L} & 0 & 0 & \frac{f_{2} v_{R 1}}{2} & 0 & \frac{f_{2} u_{1}}{2} & 0 \\ \hline 0 & 0 & 0 & 0 & M_{R} & -\frac{g_{R} u_{1}}{\sqrt{2}} & \frac{g_{R} u_{4}}{\sqrt{2}} & \frac{g_{R} v_{R 1}}{\sqrt{2}} & -\frac{g_{R} v_{R 2}}{\sqrt{2}} \\ 0 & -\frac{g_{L} u_{1}}{\sqrt{2}} & 0 & \frac{f_{2} v_{R 1}}{2} & -\frac{g_{R} u_{1}}{\sqrt{2}} & 0 & -\mu_{\Delta} & \frac{f_{2} v_{L 2}}{2} & 0 \\ 0 & \frac{g_{R} u_{4}}{\sqrt{2}} & \frac{f_{1} v_{R 2}}{2} & 0 & \frac{g_{R} u_{4}}{\sqrt{2}} & -\mu_{\Delta} & 0 & 0 & \frac{f_{1} v_{L 1}}{2} \\ -\frac{g_{1} v_{R 1}}{\sqrt{2}} & 0 & 0 & \frac{f_{2} u_{1}}{2} & \frac{g_{R} v_{R 1}}{\sqrt{2}} & \frac{f_{2} v_{L 2}}{2} & 0 & 0 & -\mu_{R} \\ \frac{g_{1} v_{R 2}}{\sqrt{2}} & 0 & \frac{f_{1} u_{4}}{2} & 0 & -\frac{g_{R} v_{R 2}}{\sqrt{2}} & 0 & \frac{f_{1} v_{L 1}}{2} & -\mu_{R} & 0\end{array}\right)$. 
As mentioned in Sect. 4.3, this is also not the full matrix, but some elements are already decoupled from this matrix. It is important to note that $\tilde{B}$ is not the MSSM $U(1)_{Y}$ bino, instead is a $S U(2)_{R} \times U(1)_{Y}$. But a linear combination of $\tilde{B}$ with $\tilde{W}_{R}$ in the limit $M_{B} \simeq M_{R}$ makes it a MSSM bino.

Defining the ratios $R_{W}=v_{L} / v_{\mathrm{SM}}=\sqrt{\frac{1-2 s_{W}^{2}}{1-s_{W}^{2}}}=0.837$ and $\tan \beta_{L}=v_{L 2} / v_{L 1}$, we can rewrite the $4 \times 4$ upper left matrix in (19), with the basis $\left\{\tilde{B}, \tilde{W}, \tilde{\phi}_{L 1}, \tilde{\phi}_{L 2}\right\}$ (where $\tilde{W}=$ $\left.\frac{\tilde{W}_{L}}{R_{W}}\right)$ as
The chargino mass matrices are

$$
M_{1 \chi^{ \pm}}=\left(\begin{array}{llllll}
M_{L} & 0 & \frac{g_{L} v_{L 2}}{2} & \frac{g_{L} u_{4}}{2} & 0 & 0 \\
0 & M_{R} & 0 & \frac{g_{R} u_{4}}{2} & \frac{g_{R} v_{R 2}}{2} & 0 \\
\frac{g_{L} v_{L 1}}{2} & 0 & \mu_{L} & -f_{1} v_{R 2} & 0 & 0 \\
\frac{g_{L} u_{1}}{2} & \frac{g_{R} u_{1}}{2} & -f_{2} v_{R 1} & \mu_{\Delta} & 0 & 0 \\
0 & \frac{g_{R} v_{R 1}}{2} & 0 & 0 & \mu_{R} & -f_{2} v_{L 2} \\
0 & 0 & 0 & 0 & -f_{1} v_{L 1} & \mu_{\Delta}
\end{array}\right) \text {, }
$$

$M_{\chi_{M S S M}^{0}}=\left(\begin{array}{cccc}M_{B} & 0 & -M_{Z} * s_{W} * c_{\beta L} & M_{Z} * s_{W} * s_{\beta L} \\ 0 & \frac{M_{L}}{R_{W}^{2}} & M_{Z} * c_{W} * c_{\beta L} & -M_{Z} * c_{W} * s_{\beta L} \\ -M_{Z} * s_{W} * c_{\beta L} & M_{Z} * c_{W} * c_{\beta L} & 0 & -\mu_{L} \\ M_{Z} * s_{W} * s_{\beta L} & -M_{Z} * c_{W} * s_{\beta L} & -\mu_{L} & 0\end{array}\right)$.

This is exactly the MSSM neutralino mass matrix, where $M_{B}=M_{1}, \mu_{L}=\mu, \beta_{L}=\beta$, and $\frac{M_{L}}{R_{W}^{2}} \simeq 1.43 M_{L}=M_{2}$.

The rest of the Higgsinos in the basis, $\left(\tilde{\Psi}_{2}{ }^{0}\right)^{T}=$ $\left\{\eta_{L 1}^{\tilde{0}}, \eta_{L 2}^{\tilde{0}}, \tilde{s}_{3}^{0}\right\}, \quad\left(\tilde{\Psi}_{3}{ }^{0}\right)^{T}=\left\{\eta_{R 1}^{\tilde{0}}, \eta_{R 2}^{\tilde{0}}\right\}$, and $\left(\tilde{\Psi}_{4}{ }^{0}\right)^{T}=$ $\left\{\delta_{12}^{\tilde{0}}, \delta_{21}^{\tilde{0}}\right\}$ are

$$
\begin{aligned}
M_{H 2} & =\left(\begin{array}{ccc}
0 & -\mu_{L 2} & f_{10} v_{L 2} \\
-\mu_{L 2} & 0 & f_{9} v_{L 1} \\
f_{10} v_{L 2} & f_{9} v_{L 1} & -\mu_{s 3}
\end{array}\right), \\
M_{H 3} & =\left(\begin{array}{cc}
0 & -\mu_{R 2} \\
-\mu_{R 2} & 0
\end{array}\right), \\
M_{H 4} & =\left(\begin{array}{cc}
0 & -\mu_{\Delta} \\
-\mu_{\Delta} & 0
\end{array}\right)
\end{aligned}
$$

In the following basis:

$$
\begin{aligned}
& \left(\Psi_{1}^{+}\right)^{T}=\left\{\imath \tilde{W}_{L}^{+}, \imath \tilde{W}_{R}^{+}, \tilde{\phi_{L 2}^{+}}, \tilde{\delta_{22}^{+}}, \tilde{\phi_{R 2}^{+}}, \tilde{\delta_{12}^{+}}\right\} \\
& \left(\Psi_{1}^{-}\right)^{T}=\left\{\imath \tilde{W}_{L}^{-}, \imath \tilde{W}_{R}^{-}, \tilde{\phi_{L 1}^{-}}, \tilde{\delta_{11}^{-}}, \tilde{\phi_{R 1}^{-}}, \tilde{\delta_{21}^{-}}\right\} \\
& \left(\Psi_{2}^{+}\right)^{T}=\left\{\tilde{\eta}_{L 2}^{+}, \tilde{\eta}_{R 2}^{+}, \zeta_{2}^{+}\right\} \\
& \left(\Psi_{2}^{-}\right)^{T}=\left\{\tilde{\eta}_{L 1}^{-}, \tilde{\eta}_{R 1}^{-}, \zeta_{1}^{-}\right\},
\end{aligned}
$$

$$
M_{2 \chi^{ \pm}}=\left(\begin{array}{ccc}
\mu_{L 2} & f_{3} u_{1} & f_{5} v_{L 1} \\
f_{4} u_{4} & \mu_{R 2} & f_{6} v_{R 1} \\
-f_{7} v_{L 2} & -f_{8} v_{R 2} & \mu_{s 12}
\end{array}\right) \text {. }
$$

\section{References}

1. E. Ma, Phys. Rev. D 73, 077301 (2006)

2. J. Kubo, E. Ma, D. Suematsu, Phys. Lett. B 642, 18 (2006)

3. E. Ma, Mod. Phys. Lett. A 21, 1777 (2006)

4. E. Ma, Ann. Fond. Broglie 31, 285 (2006)

5. E. Ma, U. Sarkar, Phys. Lett. B 653, 288 (2007)

6. E. Ma, Phys. Lett. B 662, 49 (2008)

7. E. Ma, Phys. Lett. B 659, 885 (2008)

8. E. Ma, D. Suematsu, Mod. Phys. Lett. A 24, 583 (2009)

9. E. Ma, Phys. Rev. D 80, 013013 (2009)

10. E. Ma, Phys. Lett. B 671, 366 (2009)

11. E. Ma, Phys. Lett. B 717, 235 (2012)

12. S. Khalil, H.-S. Lee, E. Ma, Phys. Rev. D 79, 041701(R) (2009)

13. E. Ma, Phys. Rev. D 79, 117701 (2009)

14. A. Aranda, J.L. Diaz-Cruz, J. Hernandez-Sanchez, E. Ma, Phys. Rev. D 81, 075010 (2010)

15. S. Khalil, H.-S. Lee, E. Ma, Phys. Rev. D 81, 051702(R) (2010)

16. E. Ma, Phys. Rev. D 81, 117703 (2010)

17. E. Ma, Phys. Rev. D 85, 091701(R) (2012)

18. Q.-H. Cao, E. Ma, J. Wudka, C.-P. Yuan, arXiv:0711.3881 [hep-ph]

19. ATLAS-CONF-2013-017, http://cds.cern.ch/record/1525524 
20. A. Belyaev, N.D. Christensen, A. Pukhov, Comput. Phys. Commun. 184, 1729 (2013). arXiv:1207.6082 [hep-ph]

21. H.L. Lai et al., CTEQ Collaboration, Eur. Phys. J. C 12, 375 (2000). arXiv:hep-ph/9903282

22. G. Hinshaw et al., [WMAP Collaboration], arXiv:1212.5226 [astro-ph.CO]

23. P.A.R. Ade et al., [Planck Collaboration], arXiv:1303.5076 [astroph.CO]

24. G. Belanger, F. Boudjema, A. Pukhov, A. Semenov, Comput. Phys. Commun. 180, 747 (2009). arXiv:0803.2360 [hep-ph]

25. K. Griest, D. Seckel, Phys. Rev. D 43, 3191 (1991)

26. A. Birkedal-Hansen, B.D. Nelson, Phys. Rev. D 64, 015008 (2001). arXiv:hep-ph/0102075

27. T. Moroi, L. Randall, Nucl. Phys. B 570, 455 (2000). arXiv:hep-ph/9906527

28. H. Baer, A. Mustafayev, E.-K. Park, S. Profumo, JHEP 0507, 046 (2005). arXiv:hep-ph/0505227

29. H. Baer, T. Krupovnickas, A. Mustafayev, E.-K. Park, S. Profumo, X. Tata, JHEP 0512, 011 (2005). arXiv:hep-ph/0511034

30. U. Chattopadhyay, D. Das, P. Konar, D.P. Roy, Phys. Rev. D 75, 073014 (2007). arXiv:hep-ph/0610077

31. T. Moroi, M. Nagai, M. Takimoto, JHEP 1307, 066 (2013). arXiv:1303.0948 [hep-ph]
32. G. Kane, R. Lu, S. Watson, Phys. Lett. B 681, 151 (2009). arXiv:0906.4765 [astro-ph.HE]

33. S. Mohanty, S. Rao, D.P. Roy, Int. J. Mod. Phys. A 27(6), 1250025 (2012). arXiv:1009.5058 [hep-ph]

34. J. Hisano, S. Matsumoto, M.M. Nojiri, O. Saito, Phys. Rev. D 71, 015007 (2005). arXiv:hep-ph/0407168

35. J. Hisano, K. Ishiwata, N. Nagata, Phys. Lett. B 690, 311 (2010). arXiv: 1004.4090 [hep-ph]

36. T. Cohen, M. Lisanti, A. Pierce, T.R. Slatyer, arXiv:1307.4082 [hep-ph]

37. S. Eidelman et al., Particle Data Group Collaboration, Phys. Lett. B 592, 1 (2004)

38. A.H. Chamseddine, R. Arnowitt, P. Nath, Phys. Rev. Lett. 49, 970 (1982)

39. R. Barbieri, S. Ferrara, C.A. Savoy, Phys. Lett. B 119, 343 (1982)

40. L.J. Hall, J. Lykken, S. Weinberg, Phys. Rev. D 27, 2359 (1983)

41. P. Nath, R. Arnowitt, A.H. Chamseddine, Nucl. Phys. B 227, 121 (1983)

42. N. Ohta, Prog. Theor. Phys. 70, 542 (1983) 